INGENIERÍA QUÍMICA

\title{
Obtención de 2-Fenil lepidinas durante la alquilación de Friedel-Crafts asistida por microondas de $N$-( $\alpha$-alilbencil) anilinas soportadas en sílica-ácido sulfúrico
}

CHEMICAL ENGINEERING

\section{2-Phenyl lepidines preparation through microwave assisted Friedel-Crafts alkylation of $\mathrm{N}$-( $\alpha$-allylbenzyl) anilines supported on silica-sulfuric acid}

\author{
Diego A. Roa, Juan Manuel Urbina*§ \\ *Escuela de Química, Universidad Industrial de Santander \\ sjurbina@uis.edu.co,droa77@gmail.com
}

(Recibido: Diciembre 6 de 2011 Aceptado: Abril 4 de 2013)

\begin{abstract}
Resumen
Se empleó el soporte ácido Sílica-Ácido Sulfúrico (SSA) para la síntesis asistida por microondas y libre de solventes de tres ejemplos de 2-fenil lepidinas (2-fenil-4-metilquinolinas) sustituidas en la posición C-6 (H, Me, OMe). La "reacción en cascada" realizada procede mediante una alquilación de Friedel-Crafts de las $N$-( $\alpha$-alilbencil)anilinas y la oxidación debida al SSA de las 2-fenil-1,2,3,4-tetrahidrolepidinas intermediarias (no aisladas), con buenos rendimientos y tiempos cortos de reacción. Los productos preparados por la metodología aquí descrita en una sola etapa fueron idénticos a los preparados anteriormente en nuestro grupo de investigación mediante reacciones sucesivas.
\end{abstract}

Palabras clave: Sílica ácido sulfúrico, síntesis asistida por radiación de microondas, 2-fenil lepidinas.

\begin{abstract}
Silica sulfuric acid (SSA) has been efficiently used as solid support in the solvent free microwave assisted synthesis of 2-phenyl lepidines (2-phenyl-4-methylquinolines) from $N$-( $\alpha$-allylbenzyl)anilines. Reactions proceed through a two step cascade involving a Friedel-Crafts alkylation + oxidation of the formed 2-phenyltetrahydrolepidines. Good to high reaction yields and short reaction times were observed. Prepared products were identical to the ones already prepared in our lab through a two step synthesis.
\end{abstract}

Keywords: Microwave assisted organic synthesis; silica sulfuric acid; 2-phenyl lepidine. 


\section{Introducción}

El fragmento de la lepidina (4-metilquinolina) aparece en estructuras con diversa actividad farmacológica y en un amplio número de moléculas con sistemas $\pi$ conjugados, aportando excelentes propiedades electrónicas / optoelectrónicas y mecánicas que incentivan el desarrollo de nuevas estrategias para su síntesis. Se han desarrollado varios métodos para la preparación de quinolinas y lepidinas, pero en su mayoría usan reactivos nocivos, largos tiempos de reacción o generan productos secundarios. Muchas de estas metodologías utilizan comúnmente una amplia variedad de catalizadores ácidos (de Lewis o de Brønsted) que son corrosivos y que producen usualmente una cantidad significativa de desechos y productos colaterales, Joule \& Mills (2000), Sridharan et al. (2011).

La experiencia previa de nuestro grupo de investigación ha mostrado la eficiencia de la alquilación intramolecular tipo FriedelCrafts para preparar diversas 2-fenil-1,2,3,4tetrahidrolepidinas a partir de $N$-( $\alpha$-alilbencil $)$ anilinas por medio de reacciones en fase homogénea, Vargas et al. (2003). Vale recalcar que el uso de soportes sólidos que promuevan la ciclación intramolecular de Friedel-Crafts asistida por radiación de microondas no ha sido ampliamente estudiado, Kurteva et al. (2004).

Al estudiar la reacción de $N$-( $\alpha$-alilbencil $)$ anilinas 1a-c adsorbidas en el soporte sólido ácido súlica-ácido sulfúrico (SSA por su sigla en inglés "Silica Sulfuric Acid") mediante radiación por microondas para la preparación de las 2-fenil-1,2,3,4-tetrahidrolepinas 2a-c, se observó la oxidación inesperada de las 2-fenil1,2,3,4-tetrahidrolepidinas 2a-c a las 2-fenil lepidinas 3a-c (Figura 1). Los productos 3a-c se obtuvieron con buenos rendimientos (70 - 90\%). Esta reacción destaca su importancia al darse bajo irradiación con microondas en una sola etapa y no emplear disolventes, contemplando dos de los doce postulados de la química verde, Sheldon et. al (2007). Además, los compuestos sintetizados son importantes precursores para la preparación de complejos metálicos derivados de 8-aminoquinolinas, empleados principalmente en electrónica orgánica.

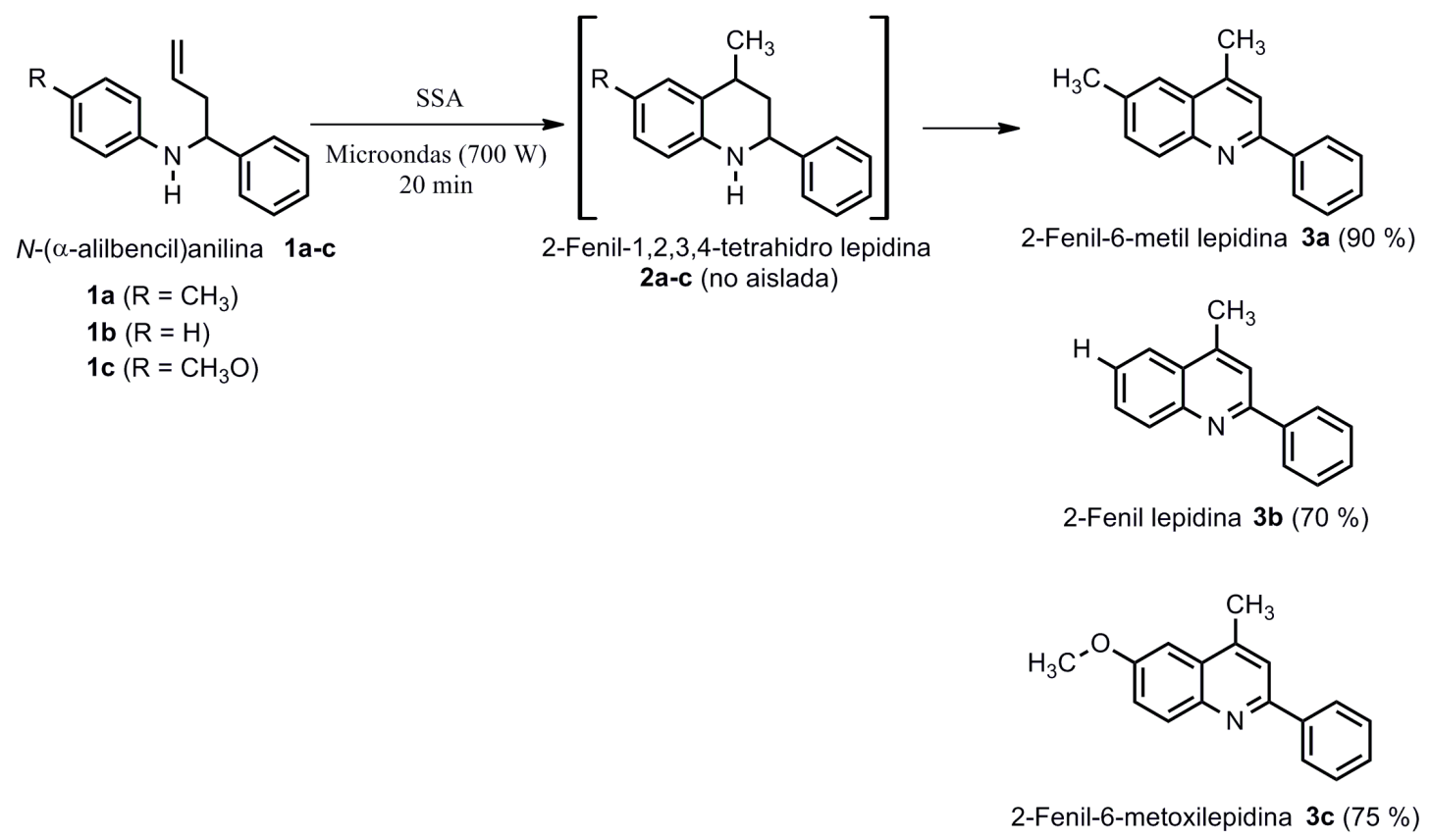

Figura 1. Formación de2-fenil lepidinas 3a-c a través de la generación de las2-fenil-1,2,3,4-tetrahidrolepidinas (no aisladas) $2 a-c$. 


\section{Metodología}

Elsoportesólidoácido, sílica-ácidosulfúrico(SSA) se preparó según el procedimiento reportado en la literatura, Zolfigol (2001), empleando un balón de fondo redondo y un embudo de adición con regulación de presión desde el cual se vertió gota a gota el ácido clorosulfónico sobre la sílica gel; el SSA se caracterizó por espectroscopía infrarroja y se determinó el total de equivalentes de ácido por gramo de soporte sólido mediante neutralización, usando $\mathrm{NaOH}$ 0,14 N; el valor resultante fue de 6 mmol de ácido por gramo de soporte sólido, valor comparable con el reportado en la literatura; vale aclarar que al tener valores semejantes de acidez, no se evaluaron ni contrastaron con la literatura otras características como el área superficial o el volumen de poro para el SSA preparado.

Las reacciones se llevaron a cabo empleando un horno de microondas doméstico multimodal (marca Panasonic, modelo "The Genius", potencia de $700 \mathrm{~W}$ ) e iniciaron con la irradiación de la $N$-( $\alpha$-alilbencil)-4-toluidina 1a adsorbida en SSA; el control del avance de la reacción se realizó mediante el monitoreo del consumo total del sustrato por cromatografía en capa delgada (CCD - cromatofolios de silufol UV245 eluyendo con mezclas de $n$-heptano y AcOEt de distinta polaridad) y cromatografía de gases (GC - Perkin Elmer AutoSystem XL acoplado a un detector FID, columna Perkin Elmer de 25 m, 5\% metil fenil silicona, diámetro interno $0.32 \mathrm{~mm}$ y espesor de 0.25 micras); una vez agotado el sustrato de partida se dio por terminado el período de la irradiación y la muestra se retiró del soporte mediante desorción empleando un sistema bifásico compuesto por una solución diluida de $\mathrm{NaHCO}_{3}$ $(\mathrm{pH}=10)$ y AcOEt, en un embudo de extracción o empleando un extractor líquido-líquido.

La reacción posteriormente fue reproducida a escala de $3 \mathrm{~g}$ de soporte sólido ácido y se obtuvo suficiente material para su caracterización mediante espectroscopía IR [Bruker Tensor 27 FTIR], cromatografía de gases acoplada a espectrometría de masas (GC-MS) [GC HP 5890A serie II, acoplado a un detector selectivo de masas
HP 5972] y resonancia magnética nuclear $\left({ }^{1} \mathrm{H}-\right.$ RMN y ${ }^{13} \mathrm{C}-\mathrm{RMN}$ ) [Bruker Avance $400 \mathrm{MHz}$ ]. El proceso fue posteriormente reproducido con éxito para la $N$-( $\alpha$-alilbencil)anilina $(1 \mathrm{~b}, \mathrm{R}=\mathrm{H})$ y para la $\mathrm{N}$-( $\alpha$-alilbencil)-4-metoxianilina $(1 \mathrm{c}, \mathrm{R}=\mathrm{OMe})$.

\section{Resultados y discusión}

El análisis comparativo del producto formado durante la irradiación de microondas de la $\mathrm{N}-(\alpha-$ alilbencil)-4-toluidina 1a adsorbida sobre el soporte sólido ácido (SSA) demostró que no se formó la 2-fenil-6-metil-1,2,3,4-tetrahidrolepidina esperada $2 \mathrm{a}$, sino su producto oxidado, la 2-fenil6-metil lepidina 3a. La lepidina 3a generada por esta inesperada reacción de oxidación fue caracterizada por IR, GC-MS y RMN ( ${ }^{1} \mathrm{H}$ y ${ }^{13} \mathrm{C}$ ); posteriormente se buscaron las condiciones para reproducir e incrementar los rendimientos del producto formado mediante un diseño experimental simple (factorial de dos niveles) variando la cantidad de muestra adsorbida sobre el SSA y el tiempo de irradiación de las microondas. Las condiciones óptimas encontradas en el diseño experimental fueron la adsorción de las $\mathrm{N}-(\alpha-$ alilbencil)anilinas 1a-c en una relación de $1 \mathrm{mmol}$ de sustrato por cada $5 \mathrm{~g}$ de SSA y su irradiación a $700 \mathrm{~W}$ por 20 minutos.

El procedimiento empleado proporcionó tres derivados de la 2-fenil lepidina 3a-c con buenos rendimientos (70-90\%), en tiempos cortos de reacción (20 $\mathrm{min}$ ) y sin la formación de productos colaterales (Figura 1). Además, se estudió el efecto de las microondas y el del agua sobre el soporte sólido ácido (SSA).

La reacción transcurre inicialmente convirtiendo la N-( $\alpha$-alilbencil)-4-toluidina 1a en la 2-fenil-6metil-1,2,3,4-tetrahidrolepidina $2 \mathrm{a}$ a través de una alquilación de Friedel-Crafts mediante la ciclación exclusiva 6-exo-trig empleando el SSA como ácido de Brønsted. Una vez formada la 2-fenil-6metil-1,2,3,4-tetrahidrolepidina 2a (observada en el seguimiento de la reacción por GC, ver Figura 2), esta se oxida a la lepidina 3 a en las condiciones de la reacción, según el análisis de GC-MS $(\mathrm{R}=\mathrm{Me}$ con $\mathrm{m} / \mathrm{z}=233$ para $3 \mathrm{a}$, en lugar de $\mathrm{m} / \mathrm{z}=237$ que 
corresponde para 2a, ver Figura 3). La oxidación de las tetrahidrolepidinas $2 \mathrm{a}-\mathrm{c}$, se presume, es asistida por la activación vía microondas del soporte sólido ácido y la reducción de los grupos sulfónicos del SSA durante la irradiación, después de cumplir su función como ácido de Brønsted en la etapa de la ciclación. Los cromatogramas no mostraron productos secundarios en la reacción, incluso al examinar una alícuota de control después de 5 minutos de irradiación (Figura 2). Cuando no se empleó la irradiación de microondas y se repitió la reacción bajo calentamiento convencional (por inmersión del reactor en aceite a $75{ }^{\circ} \mathrm{C}$ y realizando el seguimiento cada 8 horas hasta 24 horas) se observó en GC, además del producto de partida, una pequeña cantidad de la 2-fenil-6-metil-1,2,3,4-tetrahidrolepidina 2a; en ningún caso se observó el producto de oxidación 3a, lo que evidenció la importancia del efecto de microondas para que ocurriera la oxidación.

Las 2-fenil lepidinas 3a-c provenientes de la reacción fueron inesperadas, considerando que las 2-fenil-1,2,3,4-tetrahidrolepidinas 2a-c ya se habían preparado previamente en nuestro grupo de investigación empleando $\mathrm{H}_{2} \mathrm{SO}_{4}$ a $75^{\circ} \mathrm{C}$, Vargas et al. (2003). Las 2-fenil lepidinas 3a-c también habían sido preparadas en nuestro laboratorio mediante la oxidación con diclorodicianoquinona (DDQ) de las 2-fenil-1,2,3,4-tetrahidrolepidinas 2a-c, eidentificadas plenamente en investigaciones anteriores, Urbina et. al (2000); estas últimas se compararon por cromatografía de gases y espectrometría de masas con los productos provenientes del reactor irradiado con microondas y coincidieron en su totalidad.

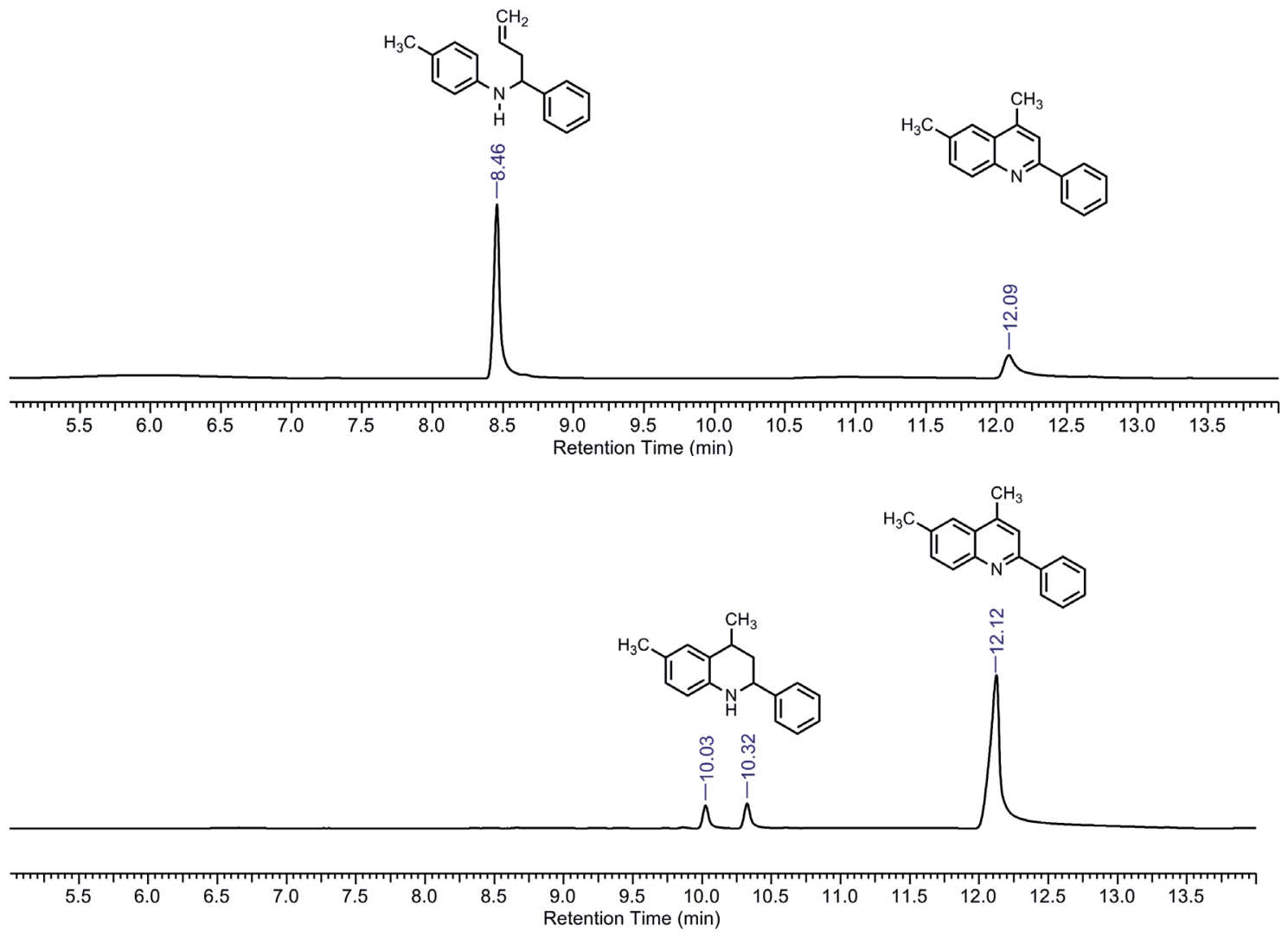

Figura 2. Cromatograma de gases (crudo de la reacción), después de varios minutos de irradiación de la $N$-( $\alpha$-alilbencil)-4metilanilina 1 a $(R=M e)$. Arriba: Después de 5 min de irradiación se observa la $N$ - $(\alpha$-alilbencil)anilina 1 a (8,5 min)y la lepidina 3 a (12,1 min). Abajo:Después de 10 min. de irradiación se observa la 1,2,3,4-tetrahidrolepidina 2 a (mezcla de diastereoisómeros a 10,0 y 10,3 min) y la lepidina 3 a $(12,1 \mathrm{~min})$. 

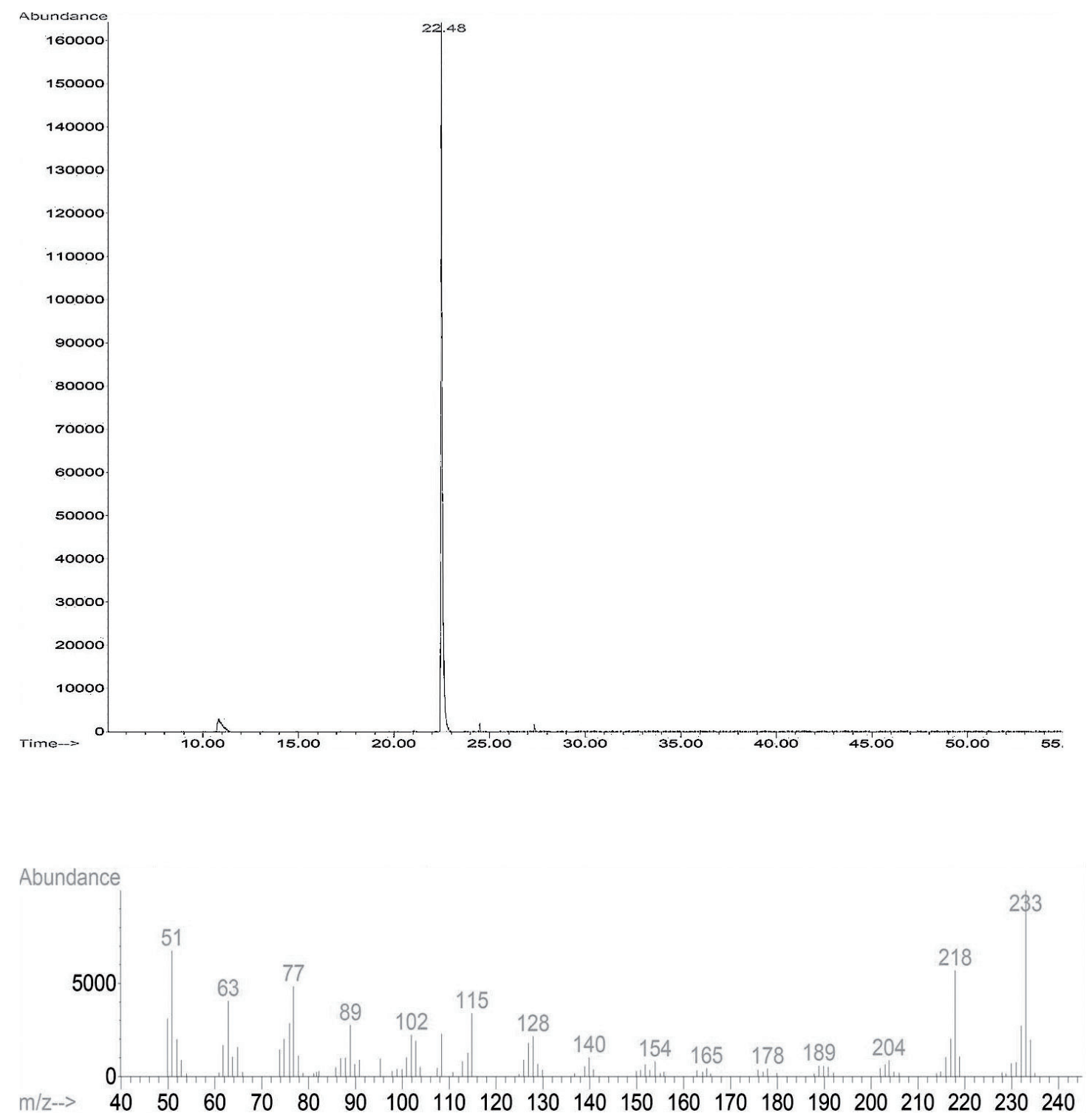

Figura 3. Cromatograma de gases del producto desorbido del soporte sin previa purificación y espectro de masas de la 2-fenil6-metil lepidina $3 a(R=M e)$.

La Figura 4 muestra el espectro IR del soporte sólido antes y después de la reacción; en el espectro IR de la SSA usada (espectro inferior) las bandas $v_{12} \mathrm{~S}-(\mathrm{OH})\left(887 \mathrm{~cm}^{-1}\right)$ y $\mathrm{v}_{4} \mathrm{~S}-(\mathrm{OH})\left(848 \mathrm{~cm}^{-}\right.$ $\left.{ }^{1}\right)$ desaparecen, indicando que el grupo funcional $-\mathrm{SO}_{3} \mathrm{H}$ no está presente en la sílica gel.

Es importante mencionar que cuando se emplean condiciones térmicas o en ausencia del soporte sólido ácido, las 2-fenil lepidinas 3a-c no se forman. Al estudiar la estabilidad de la sílica-ácido sulfúrico se observó que esta perdía totalmente su acidez al exponerse a prolongados periodos de tiempo de irradiación (> $10 \mathrm{~min}$. a $700 \mathrm{~W}$ ), indicando la desorción de los grupos sulfónicos inicialmente soportados sobre la matriz sólida.

Así, se puede considerar que el SSA y la radiación de microondas resultan la combinación imprescindible para la secuencia de reacciones 


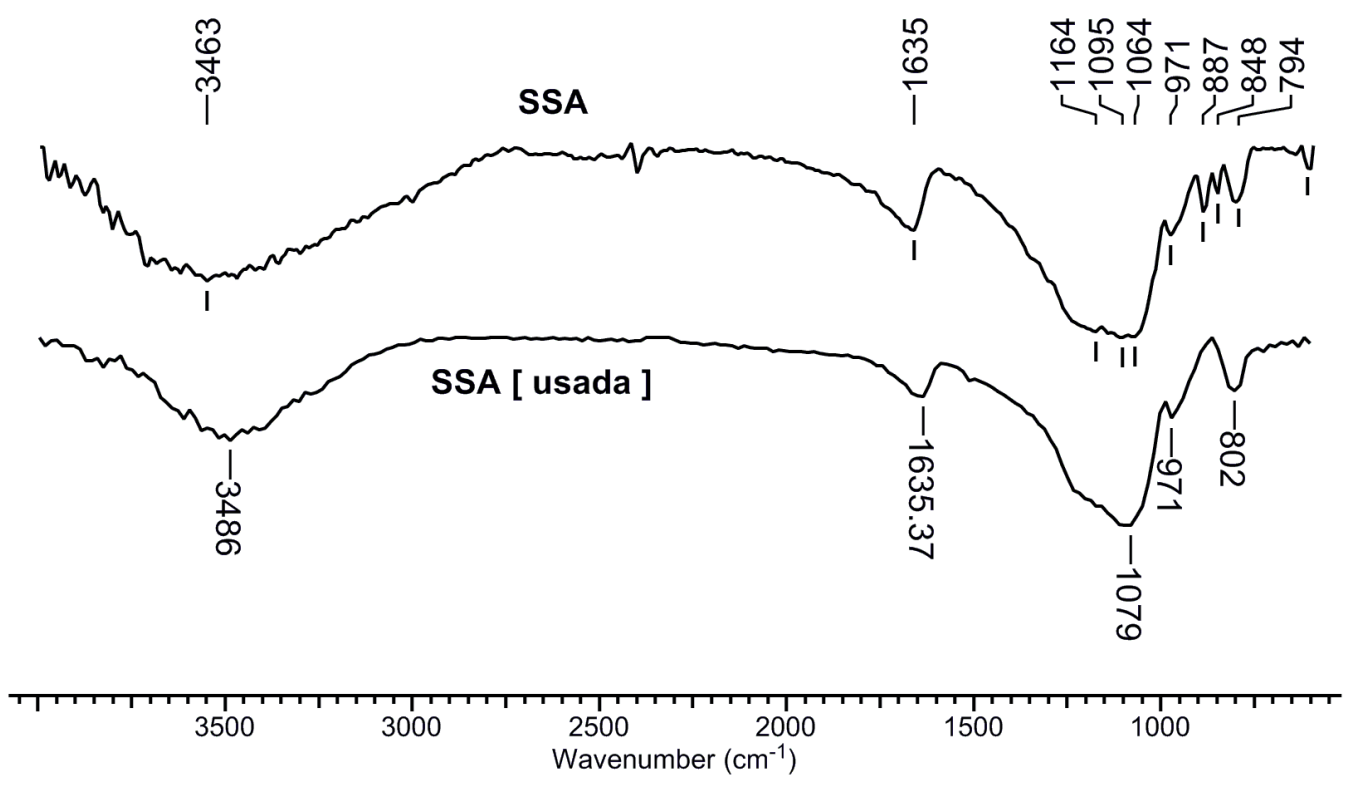

Figura 4. Espectro IR del soporte sólido ácido empleado (sílica - ácido sulfúrico, SSA) antes y después de la irradiación de microondas. Obsérvese el cambio en el perfil en la región de 890 a $840 \mathrm{~cm}^{-1}$.

ciclación + oxidación que dan lugar a la obtención de las 2-fenil lepidinas 3a-c, superando los problemas que presentan los métodos clásicos para esta reacción (productos de hidratación, manejo, seguridad, separación del producto de interés); además la metodología utilizada cumple con varios de los postulados de la química verde, como la prevención de derrames, la reducida formación de desechos al no emplear disolventes y el menor consumo energético (menos tiempo de reacción y energía focalizada a través de la irradiación de microondas), Sheldon et. al (2007). Aunque inicialmente en la investigación se planeó usar el SSA como un ácido de Brønsted alternativo para la síntesis de las 2-fenil1,2,3,4-tetrahidroquinolinas $2 \mathrm{a}-\mathrm{c}$ mediante un procedimiento similar a los previamente estudiados en nuestro grupo de investigación, el análisis de los datos obtenidos mostró que el SSA participó además en la reacción asistida por microondas como un promotor de la oxidación de las 2-fenil-1,2,3,4-tetrahidroquinolinas 2a-c a las 2-fenil lepidinas 3a-c y que una vez terminada la reacción el SSA nunca pudo recuperarse (se comprobó que después de la irradiación por microondas perdía totalmente su actividad).

\section{Conclusiones}

La alquilación de Friedel-Crafts regioselectiva 6-exo-trig y la posterior oxidación que produce las 2-fenil lepidinas $3 \mathrm{a}-\mathrm{c}$ a partir de las $N-(\alpha-$ alilbencil)anilinas 1a-c adsorbidas en sílicaácido sulfúrico durante la síntesis asistida por microondas, ocurre a través de las 2-fenil-1,2,3,4tetrahidrolepidinas $2 \mathrm{a}-\mathrm{c}$; estas fueron identificadas por GC mediante comparación con un patrón previamente preparado, pero no fueron aisladas de la masa de reacción.

La oxidación de las 2-fenil-1,2,3,4tetrahidrolepidinas $2 \mathrm{a}-\mathrm{c}$ solo se produce por el efecto de las microondas sobre el SSA presumiblemente por la reducción de los grupos sulfónicos.

No se observó la formación de productos colaterales durante el transcurso de la reacción. 
El procedimiento desarrollado presenta ventajas notables como condiciones suaves, tiempos cortos de reacción y la fácil separación de las 2-fenil lepidinas 3a-c del soporte mediante desorción con solvente.

\section{Agradecimientos}

Esta investigación fue realizada gracias al apoyo financiero de la Dirección de Investigación y Extensión de la Facultad de Ciencias - UIS, proyecto interno No. 5171. Se agradece a Sebastián Flórez por su apoyo en la discusión y revisión del documento. Diego Roa agradece a T. Ardila por el apoyo financiero para la asistencia al VII Simposio Colombiano de Catálisis SICCAT 2011.

\section{Referencias bibliográficas}

Joule, J.A. \& Mills, K. (2000). Heterocyclic Chemistry. Oxford, Blackwell Science.

Kurteva, V., Santos, A. \& Afonso, C. (2004). Microwave accelerated facile synthesis of fused polynuclear hydrocarbons in dry media by intramolecular Friedel-Crafts alkylation. Organic \& Biomolecular Chemistry 2, 514-523.
Sheldon, R.A., Arends, I. \& Hanefeld, U. (2007). Green Chemistry: Theory and Practice. Weinheim, Wiley-VCH.

Sridharan, V., Suryavanshi, P. A. \& Menéndez, J. C. (2011). Advances in the chemistry of Tetrahydroquinolines. Chemical Reviews 111, 7157-7259.

Urbina, J., Cortes, J., Palma, A., Lopez, S., Zacchino, S., Enriz, R., Ribas, J. \& Kouznetsov, V. (2000). Inhibitors of the fungal cell wall. Synthesis of 4-aryl-4-N-arylamine-1-butenes and related compounds with inhibitory activities on $\beta(1-3)$ glucan and chitin synthases. Bioorganic \& Medicinal Chemistry 8, 691-698.

Vargas, L., Castelli, M., Kouznetsov, V., Urbina, J., Lopez, S., Sortino, M., Enriz, R., Ribas, J. \& Zacchino, S. (2003). In vitro antifungal activity of new series of homoallylamines and related compounds with inhibitory properties of the synthesis of fungal cell wall polymers. Bioorganic \& Medicinal Chemistry 11, 1531-1550.

Zolfigol, M. (2001). Silica sulfuric acid/ $\mathrm{NaNO}_{2}$ as a novel heterogeneous system for production of thionitrites and disulfides under mild conditions. Tetrahedron 57, 9509-9511. 